\title{
Scénarios de régulation décentralisée des prélèvements agricoles en eau souterraine. Évaluation participative dans le bassin du Clain
}

Scenarios of irrigation groundwater decentralized management. A participative approach in the Clain basin, France

\section{Anne-Gaëlle Figureau, Marielle Montginoul and Jean-Daniel Rinaudo}

\section{OpenEdition}

\section{Journals}

Electronic version

URL: http://journals.openedition.org/economierurale/4386

DOI: 10.4000/economierurale.4386

ISSN: 2105-2581

\section{Publisher}

Société Française d'Économie Rurale (SFER)

Printed version

Date of publication: 15 July 2014

Number of pages: $27-44$

ISSN: 0013-0559

\section{Electronic reference}

Anne-Gaëlle Figureau, Marielle Montginoul et Jean-Daniel Rinaudo, « Scénarios de régulation décentralisée des prélèvements agricoles en eau souterraine. Évaluation participative dans le bassin du Clain », Économie rurale [En ligne], 342 I juillet-août 2014, mis en ligne le 15 juillet 2016, consulté le 01 mai 2019. URL : http://journals.openedition.org/economierurale/4386 ; DOI : 10.4000/ economierurale.4386 


\title{
Scénarios de régulation décentralisée des prélèvements agricoles en eau souterraine Évaluation participative dans le bassin du Clain
}

\author{
Anne-Gaëlle FIGUREAU • IRSTEA, UMR G-EAU, Montpellier ; BRGM, Service Eau, Montpellier ; \\ a.figureau@brgm.fr \\ Marielle MONTGINOUL • IRSTEA, UMR G-EAU, Montpellier \\ Jean-Daniel RINAUDO • BRGM, Service Eau, Montpellier
}

La loi sur l'eau de 2006 prévoit l'établissement d'Organismes uniques de gestion collective (OUGC) chargés de répartir la ressource en eau entre irrigants. Ceci impose de réfléchir à des modes de gestion décentralisée de l'eau d'irrigation. Les auteurs ont élaboré deux instruments économiques de gestion décentralisée respectant les principes d'Ostrom (1990) : un système de bonus-malus individuels et monétaires, et un système d'autorégulation imbriquée. Par une méthode de prospective participative, ces instruments ont été discutés avec trois groupes d'irrigants et de représentants institutionnels du bassin du Clain (86). Le premier a été mal reçu, les participants rejetant le principe " de la carotte et du bâton ». Le second a été fortement apprécié et semble constituer un futur envisageable.

MOTS-CLÉS : eau souterraine, gestion, autorégulation, bonus-malus, bassin du Clain

\section{Scenarios of irrigation groundwater decentralized management. A participative approach in the Clain basin, France}

The French Water Law of 2006 requires the establishment of local organisms for irrigation water management, in charge of sharing water among farmers. This raises questions of designing a decentralized water management. Two economic tools are designed here to comply with Ostrom's design principles (1990): a payment-and-penalty mechanism, and a system of nested self-regulation. Through a participative and foresight approach, these tools have been discussed by three groups of farmers and institutional representatives in the Clain river basin (Western France). The former was unpopular for participants, who rejected the "carrot and stick" principle. The latter has been appreciated, even judged as a possible future (JEL: Q25, Q15, Q28, Q54, K32, H39)

KEYWORDS: groundwater, management, self regulation, reward-penalty system, Clain basin

$\mathbf{E}^{\mathrm{n}}$ France, le développement peu contrôlé de l'irrigation agricole à partir des eaux souterraines fait émerger, dès les années 1990, un problème de surexploitation des aquifères, qui se manifeste dans des contextes aussi variés que le littoral languedocien, le bassin parisien ou la région PoitouCharentes. Jusque dans les années 2000, la gestion de la ressource y reste très centralisée, l'allocation, le contrôle et la sanction restant du ressort des services de l'État.

Ce contexte évolue significativement en 2006 avec la publication de la loi $\mathrm{n}^{\circ}$ 2006-1772 du 30 décembre 2006 sur l'eau et les milieux aquatiques (LEMA) qui engage un processus de décentralisation de la gestion de l'eau d'irrigation. L'État délègue la responsabilité du partage de la ressource à un Organisme unique de gestion collective (OUGC) regroupant tous les agriculteurs du bassin. Cependant, la mise en place des OUGC s'avère longue et difficile. Notamment l'élaboration d'un règlement intérieur soulève de nombreuses interrogations et pour l'OUGC qui doit à la fois rendre des comptes à l'État et 
inciter ses membres irrigants à respecter le volume prélevable, cette question est cruciale.

Cet article aborde ce problème en présentant deux mécanismes de régulation de l'usage des eaux souterraines, combinant instruments économiques et institutionnels. Le premier propose l'intégration d'outils incitatifs dans un système d'autorégulation de type OUGC; le second repose sur une autorégulation double et imbriquée, à l'image de ce qu'Ostrom (1990) appelle les nested enterprises (initiatives emboîtées) (Ostrom, 1990). Ces propositions sont ensuite confrontées aux points de vue d'acteurs de l'eau et d'agriculteurs dans le bassin du Clain (86) en région Poitou-Charentes, afin d'identifier leurs forces et faiblesses, et les conditions nécessaires à leur opérationnalité. Le cas d'étude choisi est caractérisé par une situation de déficit hydrologique chronique et le déclenchement quasi systématique de restrictions des prélèvements en été. $\mathrm{La}$ mise en débat est organisée en utilisant une méthode de prospective participative, inspirée de travaux antérieurs réalisés dans le Roussillon et en Algarve au Portugal (Rinaudo et al., 2012).

Cet article est organisé comme suit : la première partie expose la réforme de la politique de gestion de l'eau d'irrigation en France, en la replaçant dans une perspective historique ; la deuxième partie présente les deux mécanismes proposés, en précisant l'ancrage théorique ; le cas d'étude et la méthodologie déployée pour mettre en débat les propositions sont exposés dans la troisième partie ; enfin, la dernière partie et la conclusion résument les principaux résultats de ces ateliers, les apprentissages et les recommandations possibles en matière de politique publique sur ce bassin, ainsi que les perspectives de travail afin d'améliorer les scénarios.

\section{Réforme de la gestion de l'eau d'irrigation et rôle des organismes uniques}

La LEMA réforme significativement la politique de gestion de l'eau d'irrigation en France. Cette section décrit cette évolution en s'appuyant sur vingt-trois entretiens réalisés avec des experts du ministère de l'Écologie, de l'Office national de l'eau et des milieux aquatiques (ONEMA), des agences de l'eau (Loire-Bretagne, SeineNormandie, Adour-Garonne et RhôneMéditerranée et Corse) et quelques acteurs régionaux ${ }^{1}$.

\section{Un encadrement progressif des prélèvements agricoles en nappe}

En France, c'est dans un relatif vide institutionnel et donc de manière relativement incontrôlée que l'usage agricole des eaux souterraines s'est développé, représentant « une révolution silencieuse », pour reprendre les termes de Llamas et MartinezSantos (2005).

Entre 1970 et 2000, on assiste à un triplement de la surface irriguée (Gleyses, Rieu, 2004). La plupart des agriculteurs préfèrent irriguer à partir d'eaux souterraines qui représentent une ressource peu coûteuse et moins sensible aux aléas climatiques que les ressources superficielles, et qui leur permettent de gérer leur système irrigué de manière indépendante (Llamas, Martinez-Santos, 2005). Ainsi, en 2000,

1. Ces entretiens ont fait l'objet d'un rapport détaillé sur la mise en place du volet quantitatif de la loi de 2006. Pour cela, voir Figureau (2012). Les acteurs régionaux rencontrés sont la Chambre départementale d'agriculture de la Vienne (CDA 86), la Direction départementale des territoires (DDT86), le Conseil général de la Vienne (CG 86), la Délégation locale de l'agence de l'eau, la Fédération départementale de pêche et de protection des milieux aquatiques (FDPPMA86), l'Association départementale des irrigants de la Vienne (ADIV), l'association des Sources du Bé et cinq agriculteurs irrigants. 
les exploitations françaises équipées d'au moins un forage représentent $34 \%$ des exploitations équipées ; plus de la moitié de la surface irriguée est alors arrosée grâce à un forage (Gleyses, Rieu, 2004).

Mais avec l'émergence de problèmes environnementaux liés au développement de l'exploitation agricole des eaux souterraines (baisse des niveaux des nappes entraînant des étiages sévères des cours d'eau associés), la question s'est progressivement installée dans l'agenda politique. Les préoccupations environnementales ont été intégrées dans la législation française sur l'eau dès 1984, avec la loi pêche $(1984)^{2}$, et sont à l'origine de la Directive cadre sur l'eau (DCE, 2000/60/CE) du 23 octobre 2000 établissant un cadre pour une politique communautaire dans le domaine de l'eau (Parlement européen et Conseil de l'Union européenne, 2000) et de la loi sur l'eau et les milieux aquatiques (LEMA, 2006).

\section{Une gestion décentralisée des prélèvements agricoles difficile à instaurer}

La LEMA introduit dans son article 21 la nécessité de " délimiter des périmètres à l'intérieur desquels les autorisations de prélèvement d'eau pour l'irrigation sont délivrées à un organisme unique pour le compte de l'ensemble des préleveurs irrigants ». Les textes législatifs associés (ministère de l'Écologie, 2007, 2008, 2012) ont permis de préciser le système envisagé. Chaque bassin caractérisé par un déficit chronique doit faire l'objet d'une étude visant à déterminer le volume réellement disponible pour les activités humaines, dit « volume prélevable », qui doit ensuite être

2. La loi pêche a introduit la nécessité de respecter des débits d'étiage minimum dans les cours d'eau. Les eaux souterraines sont concernées dès lors qu'elles contribuent à en réalimenter. La loi pêche a donc indirectement contribué à créer des contraintes environnementales pour les irrigants. réparti entre les usages. Le volume alloué à l'irrigation est confié à un OUGC, chargé de le redistribuer entre les irrigants. Une structure existante ou constituée ad hoc peut soumettre sa candidature sur un périmètre qu'elle propose. Elle est jugée sur des critères tels que la cohérence hydrographique et/ou hydrogéologique du périmètre proposé, une bonne connaissance des prélèvements, sa capacité à représenter les irrigants (légitimité interne), ainsi que des relations reconnues avec l'Administration (légitimité externe). Une fois désigné, l'OUGC recueille les besoins de chaque irrigant et propose un plan de répartition du volume prélevable, en vue de se voir délivrer une autorisation unique pluriannuelle (jusqu'à 15 ans), qui se substituera à toutes les autorisations individuelles. L'OUGC renouvelle chaque année sa proposition de plan de répartition, qui doit être validée par le préfet. Depuis 2012, l'OUGC est autorisé à demander une redevance pour service rendu à ses irrigants.

Le système envisagé par la loi semble traduire une volonté de délégation des pouvoirs de l'État à la profession agricole. Cette décentralisation reste cependant maîtrisée, puisque l'État, via le préfet, reste maître des décisions importantes : désignation de l'OUGC, validation du plan de répartition, délivrance de l'autorisation unique... Ainsi, on considère parfois l'OUGC comme un simple mandataire (Bourgeois, 2011), « un hybride entre une délégation de service public et une organisation représentative des irrigants » (Lafitte et al., 2008). Le statut juridique des OUGC est actuellement l'obstacle majeur dans leur mise en place, ce qui leur vaut la qualification d' « objet juridique non identifié » (Morice, communication personnelle, 2012). Plusieurs aspects restent en effet très flous : responsabilité pénale en cas de non-respect du volume prélevable, autorité sur ses membres, validité juridique du règlement intérieur... Il n'existe à ce jour aucune expérience du fonctionnement 
d'un OUGC (25 ont été désignés, mais aucun ne fonctionne). Il existe donc une véritable attente de la part des acteurs locaux et de l'État en matière de règles de gestion à instaurer (Morice, communication personnelle, 2012).

\section{Vers un renforcement des pouvoirs de I'OUGC?}

Le processus de mise en place des OUGC prendra plusieurs années. C'est donc à partir de l'horizon 2020 que nous avons décidé de nous projeter pour concevoir des instruments de gestion. Nous présentons ici les évolutions qui sont susceptibles de se produire à cet horizon temporel, afin de restreindre le champ des possibles et de permettre de mieux définir le cadre dans lequel la gestion de l'eau s'inscrira.

- La connaissance du fonctionnement du système hydrogéologique sera plus précise, ce qui permettra de prévoir le comportement des aquifères et les volumes prélevables de façon plus fiable. L'essor des technologies de télégestion conduira à la généralisation des compteurs à télérelève permettant une acquisition automatique des données relatives aux prélèvements individuels. Cette évolution est la suite logique des efforts réalisés au niveau local par les chambres d'agriculture pour améliorer la connaissance et la quantification des prélèvements. Ainsi, on se rapproche d'une situation d'information parfaite : tous les prélèvements sont connus, suivis en temps réel et centralisés dans une banque de données, comme celle en construction au niveau national (ONEMA, BRGM, 2011).

- Concernant le contexte institutionnel, nous supposons que tous les bassins déficitaires seront dotés d'un OUGC. On peut prévoir un renforcement de ses pouvoirs, avec notamment une responsabilité pleine et entière du respect du volume prélevable et d'une mission de contrôle et de sanction. En effet, les experts s'accordent aujourd'hui à considérer que le statut juridique des OUGC est fragile et ne permettra pas d'instaurer des règles de gestion contraignantes. Le rapport Laffite (2008) propose 19 recommandations pour améliorer leur autorité, parmi lesquelles celle d' " étudier une disposition législative ou réglementaire conférant à l'organisme unique la compétence d'édicter un règlement intérieur opposable aux irrigants et de percevoir auprès d'eux une redevance pour service rendu » (recommandation $\left.n^{\circ} 10\right)$ et celle d' " étudier la possibilité de confier aux organismes uniques qui le désirent la responsabilité pleine et entière du respect de l'autorisation globale » (recommandation $\mathrm{n}^{\circ} 14$ ). Nous avons supposé ici l'application de ces recommandations.

\section{Réguler les prélèvements dans un contexte de décentralisation}

\section{Les différentes approches de la régulation des prélèvements}

Les eaux souterraines constituent l'exemple classique d'une ressource en bien commun : on ne peut ni les dévier ni les clôturer. Elles sont caractérisées par la non-exclusion (il est difficile d'en empêcher l'accès) et la rivalité entre les usagers (stock total et consommations simultanées limités). Le partage de l'eau souterraine en agriculture s'inscrit donc dans le cadre de la gestion d'un bien commun et constitue un problème d'action collective. Si l'on adopte la théorie des jeux comme cadre d'analyse, on peut supposer que tout irrigant rationnel maximisera ses propres prélèvements et comptera sur les autres pour modérer les leurs et ainsi maintenir la «production »du bien commun : en l'absence d'instrument de régulation et de coordination des acteurs, l'équilibre atteint est alors sous-optimal (Hardin, 1968).

La littérature classe généralement les instruments de régulation en trois groupes 
(Koundouri, 2004 ; Salzman, 2005 ; Theesfeld, 2010) :

- Les instruments de régulation par la quantité : normes, quotas, droits (d'usage ou de propriété) ou restrictions. Ces instruments visent à limiter directement le nombre de préleveurs ou les volumes prélevés.

- Les instruments de régulation par le prix : taxes, subventions, systèmes de tarification, ou marchés de l'eau. Ils fournissent à l'agent des incitations monétaires afin qu'il adopte le comportement souhaité : le coût d'une action indésirable est augmenté, celui d'une action désirable diminué.

- Les instruments de régulation par la persuasion (Salzman, 2005) : information, sensibilisation et éducation des agents.

Les modes de coordination correspondent à des arrangements institutionnels situés entre trois pôles : la coordination hiérarchique (command and control), la gestion décentralisée (ou auto-organisation) et la coordination par le marché. Une littérature récente s'est beaucoup intéressée aux formes de gestion décentralisée. Remettant en cause le modèle de Hardin (1968) sur l'épuisement inexorable d'une ressource en bien commun, de nombreux cas d'études, en Espagne, Inde, Pakistan, en Égypte ou au Népal, ont été rapportés, démontrant l'efficacité de l'autorégulation. Celle-ci peut être définie comme la capacité des usagers d'une ressource à « concevoir, suivre et mettre en application leurs propres règles pour maintenir un usage intensif d'une ressource commune locale » (modifié de Ostrom, Gardner, 1993).

\section{Facteurs favorables à l'autorégulation et principes d'élaboration des instruments}

Le succès de ces nouvelles formes de coordination et de régulation s'explique d'abord par une meilleure connaissance du milieu et de la ressource par les acteurs locaux, qui sont alors capables de concevoir des règles de gestion adaptées au contexte local (Lopez-Gunn, Martinez-Cortina, 2006 ; Rose, 2002 ; Van Steenbergen, 2006). L'autonomie et la confiance accordées au groupe par une autorité externe sont valorisantes et injectent une volonté de réussir dans l'organisation (Lopez-Gunn, 2003 ; VanSteenbergen, 2006). La démarche participative permet à ceux qui subissent les règles de pouvoir contribuer à leur conception : le risque d'un sentiment d'injustice envers le système est fortement réduit et les règles ont plus de chance d'être respectées (Lopez-Gunn, 2003 ; Lopez-Gunn, Martinez-Cortina, 2006 ; Vyrastekova, von Soest, 2003). Enfin, la pression sociale et le contrôle interne favorisent les comportements vertueux (Lopez-Gunn, 2003 ; VanSteenbergen, 2006).

Les systèmes autorégulés réussiront d'autant mieux que les usagers seront caractérisés par : une forte dépendance envers la ressource, une compréhension commune de son fonctionnement et de leurs interactions, un faible taux d'actualisation (valorisation des gains futurs), une confiance réciproque, une autonomie, une expérience préalable dans une organisation et un certain degré de leadership (Ostrom, 2000 ; Romagny, Riaux, 2007 ; Schlager, Lopez-Gunn, 2005 ; Theesfeld, 2010 ; Van Steenbergen, 2006).

On retrouve dans ces travaux les principes énoncés par Ostrom (1990), repris dans le tableau 1. Ces huit principes ont depuis été complétés par Ostrom elle-même et d'autres auteurs sur la base d'études de cas supplémentaires (Agrawal, 2001 ; Baland, Platteau, 1996 ; Ostrom, 2000 ; Schlager, Lopez-Gunn, 2005). L'approche en elle-même a été mise en perspective par Ruf (2011) et critiquée par Agrawal (2001).

Les OUGC pourraient, à moyen terme, s'apparenter à un mode de gestion décentralisée (voir paragraphe suivant). Nous avons donc cherché à proposer des modes 
de gestion (i) qui s'insèrent dans un contexte de gestion décentralisée, (ii) qui incitent au respect d'un volume prélevable total et (iii) dont la mise en discussion nourrit la réflexion sur l'établissement des règlements intérieurs des OUGC.

\section{Premier instrument Un système de bonus-malus}

Le premier instrument suppose que les pénalités individuelles sont plus efficaces lorsqu'elles sont couplées à des récompenses individuelles, et imposées par les pairs. Fehr et Gächter (2000) ont testé en économie expérimentale un jeu de bien public dans lequel les joueurs peuvent payer pour infliger une pénalité à ceux qu'ils jugent ne pas contribuer assez. Ce traitement montre une hausse des contributions et ce d'autant plus lorsque le joueur conserve les mêmes partenaires de jeu. Les expériences de Sefton et al. (2007) corroborent ces résultats et montrent que la menace de sanction suffit à maintenir les contributions à un niveau élevé. La pression sociale et le jugement des autres influent donc sur les comportements des agents. Dans un second traitement, Sefton et al. (2007) ont testé l'impact de la possibilité pour les joueurs de récompenser les autres. La récompense seule ne semble pas efficace ; en revanche, lorsqu'elle est couplée à la possibilité de sanctionner, les contributions et le gain global net atteignent des niveaux maximaux.

Nous avons donc conçu un système de bonus-malus, géré par l'OUGC. L'irrigant ayant dépassé son volume prélevable individuel est pénalisé financièrement par un malus proportionnel à son dépassement alors que l'irrigant ayant économisé bénéficie d'un bonus proportionnel à son économie. Les bonus sont alimentés par les malus et leurs montants, en $€ / \mathrm{m}^{3}$ économisé, sont calculés chaque année en fonction de cette recette, du nombre de bénéficiaires et de l'économie réalisée par l'agriculteur.
Le montant des malus, en $€ / \mathrm{m}^{3}$ supplémentaire prélevé, pourra être ajusté dans le temps afin de maintenir ou de faire apparaître des comportements vertueux et ainsi de respecter le volume prélevable total.

Concrètement, la mise en place d'un tel système pourrait se baser sur un montant du malus à $0,30 € / \mathrm{m}^{3}$, ce qui est, à dire d'expert, incitatif, mais pas dissuasif (c'est-à-dire inférieur à la valeur marginale, ce qui conduit à réduire le prélèvement sans nécessairement aboutir au respect du quota) dans des systèmes de production de polyculture et de production de semences. Au-delà de $30 \%$ de dépassement, le malus est doublé, passant ainsi à un niveau jugé supérieur à la valeur marginale de l'eau dans la plupart des productions de la zone.

Ce système a trois principaux avantages : - une incitation «positive » portée par la récompense s'ajoute au système classique de pénalité financière ;

- c'est un système financier fermé ;

- l'OUGC est incité à appliquer les sanctions, puisqu'elles sont nécessaires au versement des bonus.

\section{Un second instrument L'autorégulation imbriquée}

Le second système de gestion repose sur une responsabilisation des irrigants, en favorisant la mise en place de dispositifs de gouvernance emboîtés ou nested enterprises (Ostrom, 1990). Il s'agit de placer une institution intermédiaire entre les agriculteurs et l'OUGC, créant de fait deux niveaux d'autorégulation. L'OUGC offre la possibilité aux irrigants de se regrouper autour d'un contrat, appelé « contrat solidaire ». Un volume unique est attribué au collectif que ce dernier se partage librement (figure 1). Ainsi, l'OUGC ne veille plus au respect des volumes prélevables individuels, mais uniquement à celui du volume prélevable total du groupe. Le 
contrat solidaire permet donc qu'il se crée des arrangements entre irrigants afin que les dépassements réalisés par les uns soient compensés par les économies des autres.

La démarche restant volontaire, les irrigants ne souhaitant pas signer de contrat relèvent du système individuel classique (en cas de dépassement, imposition d'une pénalité individuelle proportionnelle au volume supplémentaire prélevé).

Pour inciter les irrigants à se regrouper, l'OUGC offre aux signataires du contrat une réduction de la cotisation à l'organisme. Celle-ci s' assimile à la contrainte de participation dans la théorie des contrats. Toutefois, si le volume prélevable total venait à être dépassé, la pénalité appliquée serait collective (Segerson, 1988) et d'un montant par $\mathrm{m}^{3}$ fortement supérieur à celui du système individuel classique. Cela correspond à la contrainte d'incitation de la théorie des contrats et vise à faire en sorte qu'un comportement de passager clandestin (i.e. un signataire ayant l'intention de ne pas respecter son engagement) soit plus coûteux que le choix de la stratégie individuelle. Enfin, le scénario suppose que les membres du groupe peuvent observer les comportements de leurs partenaires, par un accès, via un site internet, aux relevés de tous les compteurs d'eau du groupe.

Les avantages du contrat solidaire sont nombreux :

- La démarche n'étant pas obligatoire, elle garantit l'engagement volontaire des signataires.

- Il offre de la souplesse : les irrigants peuvent s'arranger entre eux, permettant à certains de dépasser leur allocation individuelle.

- Il permet que se crée une réelle coopération entre les irrigants, favorisée par un nombre limité de personnes et par les liens sociaux qui les unissent (Aoki, Challe, 2006).

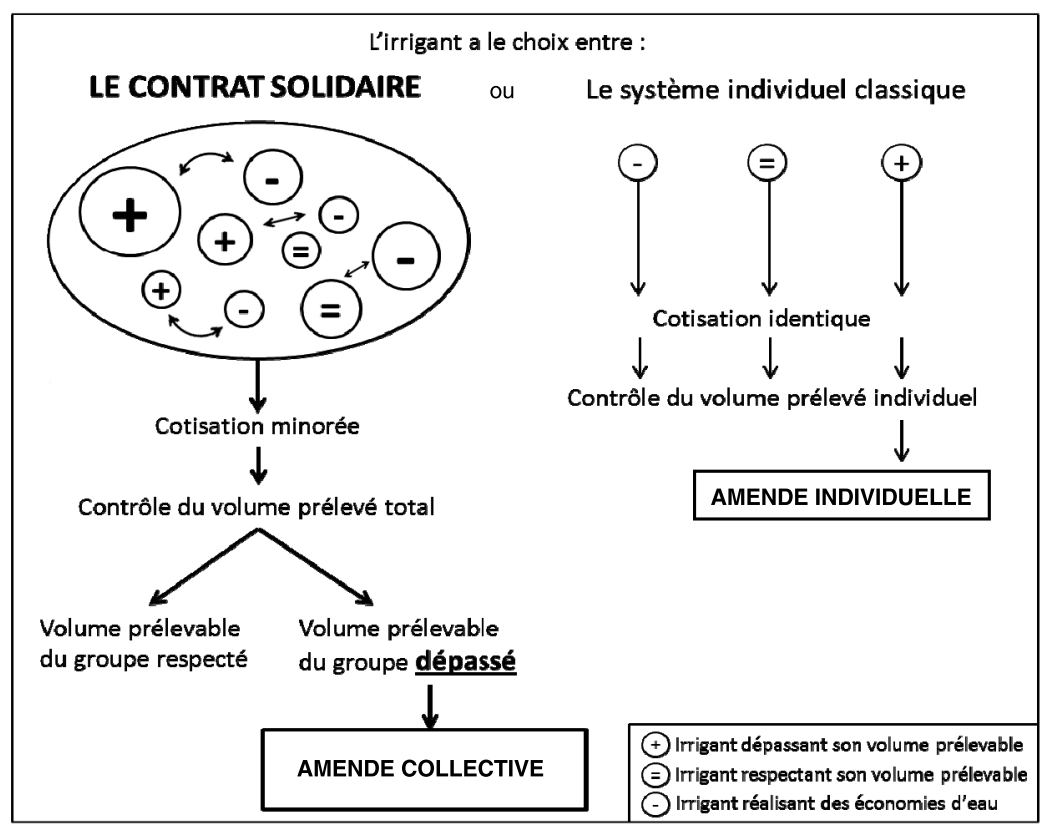

Source : les auteurs. 
- Il peut être l'occasion de faire valoir un engagement pour une irrigation raisonnée et donc restaurer l'image des irrigants auprès de la société.

- Le contrôle est réalisé par les membres et est facilité par l'accès aux données de prélèvements (compteurs à télérelève).

- Il permet de réduire le nombre d'interlocuteurs de l'OUGC et donc d'abaisser ses coûts de gestion. Le temps économisé peut être mis à profit pour un contrôle plus poussé des irrigants restés en individuel.

Les deux instruments proposés ici s'inscrivent dans une démarche d'autorégulation et respectent les principes d'Ostrom (1990) (tableau 1).
Nous avons souhaité mettre en discussion ces instruments avec les acteurs locaux et ainsi confronter notre raisonnement théorique à la réalité du terrain. La partie suivante présente la méthodologie de cette phase de test qualitatif conduite sur le bassin du Clain.

\section{Test des instruments dans le bassin du Clain}

\section{Le bassin du Clain (86)}

Notre cas d'étude concerne la partie du bassin du Clain située dans le département de la Vienne (carte 1). C'est un cas typique de surexploitation des eaux souterraines

Tableau 1. Instruments économiques imaginés et principes d'Ostrom (1990)

\begin{tabular}{|c|c|c|}
\hline PRINCIPES & [OUGC + BONUS-MALUS] & [CONTRAT SOLIDAIRE] \\
\hline $\begin{array}{l}\text { 1. Une délimitation claire de l'aire } \\
\text { géographique et des membres }\end{array}$ & $\begin{array}{l}\text { Le périmètre est fixé : celui de } \\
\text { l'OUGC. }\end{array}$ & Le périmètre est spécifié dans le contrat. \\
\hline $\begin{array}{l}\text { 2. Des règles internes fixant } \\
\text { des limites de prélèvement et } \\
\text { adaptées aux conditions locales }\end{array}$ & $\begin{array}{l}\text { L'OUGC établit un plan de réparti- } \\
\text { tion du volume total prélevable et } \\
\text { fournit une étude d'impact associée. }\end{array}$ & $\begin{array}{l}\text { Les cosignataires d'un contrat s'accordent } \\
\text { sur une répartition du volume total du } \\
\text { groupe. }\end{array}$ \\
\hline 3. Une démarche participative & $\begin{array}{l}\text { L'OUGC est doté d'une « instance de } \\
\text { consultation représentant les irri- } \\
\text { gants » afin de justifier de « l'aspect } \\
\text { collégial de la décision de réparti- } \\
\text { tion » (circulaire du } 30 \text { juin 2006) }\end{array}$ & $\begin{array}{l}\text { Tous les irrigants signataires participent } \\
\text { à la construction des règles internes. }\end{array}$ \\
\hline $\begin{array}{l}\text { 4. Un contrôle réalisé par le } \\
\text { groupe ou par des membres de } \\
\text { confiance }\end{array}$ & $\begin{array}{l}\text { L'OUGC a accès à la banque de } \\
\text { centralisation des données de prélè- } \\
\text { vements sur son périmètre. }\end{array}$ & $\begin{array}{l}\text { Les irrigants ont tous accès aux données } \\
\text { de prélèvement des autres. }\end{array}$ \\
\hline $\begin{array}{l}\text { 5. Des sanctions graduelles et } \\
\text { appliquées ou approuvées par } \\
\text { le groupe }\end{array}$ & $\begin{array}{l}\text { L'OUGC met en place un système de } \\
\text { bonus-malus qui impose des } \\
\text { pénalités proportionnelles au } \\
\text { volume prélevé supplémentaire et } \\
\text { selon des montants graduels. }\end{array}$ & $\begin{array}{l}\text { Au sein du groupe : le contrat peut inté- } \\
\text { grer des sanctions des irrigants entre eux. } \\
\text { Entre l'OUGC et le groupe: en cas de } \\
\text { dépassement du volume total, le groupe } \\
\text { est sanctionné par une pénalité collective }\end{array}$ \\
\hline $\begin{array}{l}\text { 6. Un accès rapide et peu coûteux } \\
\text { à des arènes locales de résolution } \\
\text { de conflits }\end{array}$ & $\begin{array}{l}\text { L'OUGC peut mettre en place des } \\
\text { espaces de dialogue et de résolution } \\
\text { de conflits. }\end{array}$ & $\begin{array}{l}\text { Le dialogue et la résolution des conflits } \\
\text { sont facilités par la taille limitée du } \\
\text { groupe, et la proximité sociale et } \\
\text { géographique entre les membres. }\end{array}$ \\
\hline $\begin{array}{l}\text { 7. Une reconnaissance de } \\
\text { l'organisation par l'autorité locale } \\
\text { ou nationale. }\end{array}$ & $\begin{array}{l}\text { L'OUGC est désigné par le préfet } \\
\text { et ses statuts sont officialisés par } \\
\text { décret. }\end{array}$ & $\begin{array}{l}\text { Le contrat est aussi signé par l'OUGC, } \\
\text { qui reconnaît par là le groupe. }\end{array}$ \\
\hline $\begin{array}{l}\text { 8. Une gouvernance pouvant être } \\
\text { organisée en plusieurs couches } \\
\text { en cas de ressource étendue }\end{array}$ & $\begin{array}{l}\text { À l'échelle du bassin, ce n'est pas le } \\
\text { cas dans ce système. }\end{array}$ & $\begin{array}{l}\text { Un contrat solidaire représente un } \\
\text { sous-groupe. }\end{array}$ \\
\hline
\end{tabular}




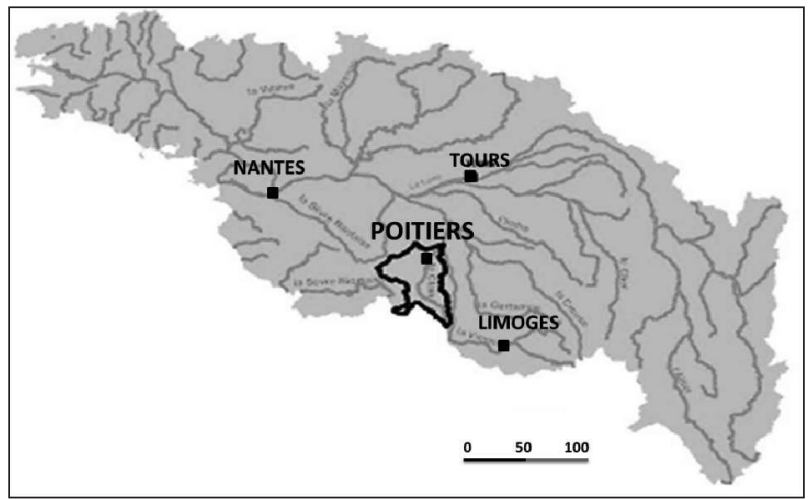

Source : les auteurs.

par l'usage agricole. Le Clain, dont le bassin s'étend sur $3200 \mathrm{~km}^{2}$, est un affluent de la Vienne et compte plusieurs aquifères superposés, dont les plus exploités sont ceux du Dogger (exploité pour l'irrigation notamment) et du Lias. Ces deux aquifères étant caractérisés par des déficits chroniques, ils sont classés en Zone de répartition des eaux $(\mathrm{ZRE})^{3}$ depuis 1994. Ils sont tous deux très sensibles aux prélèvements.

La Surface agricole utile (SAU) représente $90 \%$ du bassin du Clain, soit plus de 267000 hectares en 2000, dont $41 \%$ de céréales, $27 \%$ de surface fourragère, $11 \%$ de maïs grain, $11 \%$ de tournesol, et $9 \%$ de colza. La surface en maïs grain a augmenté de $80 \%$ de 1979 à 2000. Les surfaces irriguées ont progressé de 213 ha en 1979 à 28150 ha en 2000 (11\% de la $\mathrm{SAU})$. Les exploitations équipées représentent $15 \%$ des exploitations (656 exploitations) et irriguent surtout du maïs

3. Le classement en ZRE implique des modalités d'autorisations de prélèvement plus strictes (notamment, l'abaissement des seuils à partir desquels il est nécessaire de faire une déclaration ou demander une autorisation de prélèvement). Pour plus d'information, voir les articles R211-71 à R211-74 du Code de l'environnement. grain (13 000 ha) et des céréales à paille (6 000 ha). Les autres cultures irriguées sont les oléagineux (1 262 ha), les surfaces fourragères (1 $034 \mathrm{ha}$ ), le melon (614 ha), les protéagineux (294 ha) et le tabac (158 ha) (Conseil général de Vienne, 2011). Les prélèvements agricoles du bassin s'élèvent en moyenne à $32000 \mathrm{~m}^{3}$ par an et sont majoritairement effectués dans les eaux souterraines.

Notre intérêt pour ce terrain a également été motivé par l'existence d'un échelon institutionnel intermédiaire : les irrigants du département de la Vienne sont regroupés au sein de l'Association des Irrigants de la Vienne (ADIV 86), créée en 1973. Elle rassemble 600 adhérents dans tout le département, représentant ainsi $90 \%$ des surfaces irriguées. Dans les années 1990, celle-ci s'est associée à la Chambre départementale d'agriculture afin de mettre en place une démarche visant à améliorer les connaissances sur le fonctionnement hydrogéologique de la ressource. Les acteurs du Clain disposent ainsi d'une bonne connaissance de la ressource et des prélèvements. Dès l'an 2000, tous les forages situés en ZRE ont été équipés de compteurs et un réseau de 26 piézomètres suit 
quotidiennement la ressource souterraine sur tout le département. Le bassin du Clain a été le premier à mettre en place la gestion volumétrique, dès 1999. Les irrigants sont invités, dès le mois de décembre, à soumettre un projet d'irrigation, dans lequel ils déclarent leur projet d'irrigation (surfaces et cultures) et le volume nécessaire correspondant. Les volumes finalement alloués correspondent le plus souvent à un volume historique, même si des règles d'attribution ont pourtant été fixées (des plafonds de volumes à l'hectare par culture).

Le bassin du Clain est divisé en $14 \mathrm{sec}-$ teurs de gestion, dépendant chacun d'un piézomètre de référence, pour lequel des seuils d'alerte et de coupure sont fixés. Le dépassement du seuil d'alerte déclenche une gestion hebdomadaire avec le respect d'un Volume hebdomadaire réduit (VHR), correspondant à une réduction de $50 \%$. En cas de coupure, le prélèvement est strictement interdit. La gestion de l'eau fait l'objet d'une réflexion permanente (comité des usagers, cellule de vigilance), ce qui a été déterminant dans le choix du terrain et nous a permis de réunir sans difficulté toutes les parties concernées par la gestion de l'eau d'irrigation.

Aujourd'hui, ce système fonctionne bien, mais est appelé à supporter les réductions massives des prélèvements envisagés pour l'atteinte du volume prélevable, fixé à $17500 \mathrm{~m}^{3}$. L'effort de réduction à l'horizon 2017 s'élève donc à $15000 \mathrm{~m}^{3}$. Pour y parvenir, le « contrat territorial » adopté en 2012 par la Commission locale de l'eau (CLE) du Schéma d'Aménagement et de Gestion des Eaux (SAGE) prévoit deux types d'outils : la construction subventionnée de retenues de substitution (jusqu'à $13000 \mathrm{~m}^{3}$ ) et les mesures d'économie (3 $000 \mathrm{~m}^{3}$ ). Cependant, la construction et le fonctionnement des retenues pourraient s'avérer extrêmement coûteux, ce qui limiterait le stockage et accroîtrait d'autant l'effort nécessaire en matière d'économie d'eau. La bonne mise en œuvre du contrat passe d'abord par l'établissement d'un OUGC - pas encore désigné, la candidature de la Chambre d'agriculture étant à l'étude -, mais implique aussi de réfléchir à de nouveaux systèmes de gestion.

\section{Méthodologie déployée}

La première étape du travail (mai 2012) a consisté à réaliser une série d'entretiens individuels avec les principaux acteurs du bassin ${ }^{4}$. L'objectif était de comprendre la gestion actuelle, mais aussi d'appréhender les positions défendues par les acteurs, ainsi que les enjeux économiques et politiques sous-tendant leur positionnement. Cette étape a aussi créé un lien social avec les acteurs et motivé leur participation aux ateliers. L'information collectée a permis la contextualisation des scénarios, dont l'objectif est de démontrer aux participants que les chercheurs ont compris les enjeux techniques, économiques et politiques de la situation actuelle, et qu'ils les ont intégrés dans leur réflexion prospective. Ceci a pour but d'éviter que les participants rejettent les scénarios en les considérant comme trop théoriques ou inadaptés à la réalité de terrain. Ainsi, l'ensemble des caractéristiques locales sont intégrées aux scénarios : géographie, hydrogéologie, type d'agriculture, organisation professionnelle des irrigants... Par exemple, nous avons intégré à nos scénarios la haute sensibilité de la nappe du bassin du Clain, la gestion hebdomadaire déjà en vigueur et la construction d'une partie des retenues de substitution aujourd'hui à l'étude.

La seconde étape, conduite le mois suivant, a consisté à organiser des ateliers visant à évaluer avec les acteurs les scénarios

4. La DDT 86, la CDA 86, l'ADIV, le CG 86, le service régional du BRGM, la FDPPMA 86, le service départemental de l'ONEMA et la Délégation Poitou-Limousin de l'agence de l'eau LoireBretagne. Cinq agriculteurs ont également été rencontrés lors de cette première étape. 


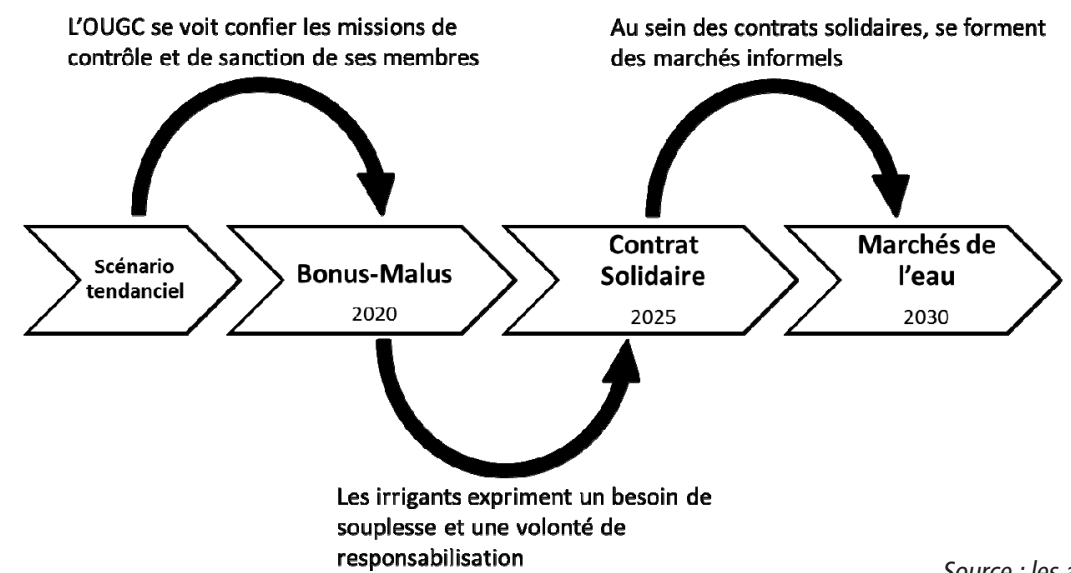

Source : les auteurs 5 .

de régulation présentés précédemment. Les scénarios ont été décrits comme une succession logique d'évènements imbriqués dans une même histoire cohérente, se déroulant entre 2020 et 2035 (figure 2). En effet, nos scénarios présentent une progression dans les choix politiques nécessaires à leur apparition. De plus, présenter les scénarios comme une succession d'évènements chronologiques vise à introduire progressivement des hypothèses restant valides pour l'ensemble des scénarios et de ne faire varier qu'un nombre limité d'éléments à chaque étape de la discussion.

Les scénarios ont été rédigés sous forme d'articles de presse pour en faciliter la lecture et susciter facilement des réactions (annexe 1). Ils ont été envoyés aux participants une semaine avant les ateliers.

Nous avons organisé 3 ateliers : un premier avec 10 institutionnels ${ }^{6}$, un deuxième

5. Nous ne discuterons pas du scénario de marché de l'eau dans cet article, le sujet méritant un traitement détaillé.

6. La DDT 86, la CDA 86 (2 personnes), l'ADIV, le CG 86, le service régional du BRGM, la FDPPMA 86, le service départemental de l'ONEMA la Délégation Poitou-Limousin de l'agence de l'eau LoireBretagne et la Direction régionale de l'environnement, de l'aménagement et du logement (DREAL). avec 15 irrigants adhérents de l'ADIV et un dernier avec 5 irrigants de l'Association des Sources du Bé. Nous avons choisi de séparer les groupes des deux associations en raison de leurs divergences d'opinion sur la gestion de l'eau du bassin, dont nous avions eu connaissance lors de la phase initiale d'entretiens. Il s'agissait d'éviter que le débat soir bloqué par des tensions interpersonnelles préexistantes et ainsi de garantir une meilleure liberté de parole et des résultats contrastés entre les deux ateliers. Le tableau 2 met en évidence les principales différences d'orientations techniques des deux groupes d'irrigants.

Chaque atelier se déroule de la même façon : après la présentation d'un scénario, illustrée par un poster, les participants sont invités à donner leur avis en renseignant un questionnaire papier, puis en l'exprimant à l'oral lors d'un tour de table, ce qui permet la mise en débat.

\section{Résultats}

Cette section expose les principaux résultats issus des ateliers organisés les 12 et 13 juin 2012 à Poitiers. Le tableau 3 synthétise l'opinion des participants, en distinguant les trois groupes. La notion 
Tableau 2. Principales caractéristiques des exploitations des groupes d'irrigants ayant participé aux ateliers

\begin{tabular}{lcc}
\hline & ADIV & Sources du Bé \\
\hline Total d'irrigants & 15 & 5 \\
Âge moyen (ans) & 46 & 48 \\
Orientation technique & Céréaliculture & Polyculture-élevage \\
SAU moyenne de l'exploitation (ha) & 204 & 111 \\
SAU irriguée moyenne de l'exploitation (ha) & 85 & 25 \\
Volume moyen attribué par exploitation (m $\left.\mathrm{m}^{3}\right)$ & 137000 & 57250 \\
Volume moyen attribué par hectare $\left(\mathrm{m}^{3} / \mathrm{ha}^{\text {irrigué})}\right.$ & 2043 & 2055 \\
\hline
\end{tabular}

Source : les auteurs.

Tableau 3. Opinion des participants concernant l'efficacité et la souhaitabilité des deux scénarios

\begin{tabular}{|c|c|c|c|c|c|c|c|c|c|c|c|c|}
\hline \multirow{3}{*}{ En \% } & \multicolumn{4}{|c|}{ EFFICACITÉ } & \multicolumn{8}{|c|}{ SOUHAITABILITÉ } \\
\hline & \multicolumn{2}{|c|}{$\begin{array}{l}\text { Bonus- } \\
\text { Malus }\end{array}$} & \multicolumn{2}{|c|}{$\begin{array}{l}\text { Contrat } \\
\text { Solidaire }\end{array}$} & \multicolumn{4}{|c|}{ Bonus-Malus } & \multicolumn{4}{|c|}{ Contrat Solidaire } \\
\hline & Oui & Non & Oui & Non & Oui & Non & NSP* & $N R * *$ & Oui & Non & NSP* & $N R * *$ \\
\hline Irrigants ADIV (15) & 13 & 87 & 87 & 13 & 7 & 80 & 13 & 0 & 93 & 0 & 7 & 0 \\
\hline Irrigants Bé (5) & 0 & 100 & 100 & 0 & 20 & 60 & 20 & 0 & 100 & 0 & 0 & 0 \\
\hline Moyenne irrigants (20) & 10 & 90 & 90 & 10 & 10 & 75 & 15 & 0 & 95 & 0 & 5 & 0 \\
\hline Institutionnels (10) & 10 & 90 & 100 & 0 & 10 & 60 & 20 & 10 & 80 & 10 & 0 & 10 \\
\hline Moyenne sur le total (30) & 10 & 90 & 93 & 7 & 10 & 70 & 17 & 3 & 91 & 3 & 3 & 3 \\
\hline
\end{tabular}

Note : * Ne sait pas ; ${ }^{* *}$ Non-réponse

Source : Les auteurs.

d'efficacité est définie comme la capacité de l'instrument à inciter au respect du volume prélevable global. Le caractère souhaitable renvoie, selon la personne, à la notion d'efficience économique, de justice distributive, de développement durable des territoires, etc.

Si un biais lié à l'aspect prospectif de la discussion est inévitable, l'effort de contextualisation des scénarios le limite en aidant les participants à s'approprier les scénarios et à se projeter dans un nouveau cadre institutionnel. Le contenu des débats nous permet aussi de nuancer les réponses au questionnaire.

\section{Le système bonus-malus Un système jugé inefficace}

Concernant le bonus-malus, $70 \%$ des participants le jugent non souhaitable, et ce pour plusieurs raisons.
Le malus : une autorisation de dépassement?

Les participants estiment les montants du malus trop peu incitatifs $\left(0,30 € / \mathrm{m}^{3}\right.$ entre 10 et $30 \%$ de dépassement, $0,60 €$ au-delà). La plupart affirment qu'à ce prix, beaucoup d'irrigants seraient prêts à payer pour sauver leur récolte : sur les 20 irrigants, 12 préféreraient payer un malus, 4 tenter d'obtenir un bonus et 6 n'ont pas répondu (la question permettait de cocher à la fois bonus et malus). Le principe du malus est critiqué, car les agriculteurs pourraient avoir le sentiment d'acheter de l'eau (acte légitime) et non plus d'enfreindre les règles. De même, payer un malus reviendrait à payer une sorte de « droit de produire », qui creuserait donc les inégalités entre les exploitations. C'est pourquoi $90 \%$ des participants pensent que ce système ne permettra pas de faire respecter le volume prélevable. 
Un bonus peu incitatif

Le bonus a lui aussi suscité des réticences. Dépendant de nombreuses variables, l'incertitude sur son montant est forte et réduit son pouvoir incitatif. Le calcul du montant par $\mathrm{m}^{3}$ apparaît comme compliqué et potentiellement opaque.

\section{Des injustices générées par le système}

Selon les irrigants, seuls ceux bénéficiant de gros volumes pourront réaliser des économies d'eau; de même, les variabilités climatiques au sein d'un même secteur généreront des injustices, puisque certaines exploitations recevront plus de précipitations et bénéficieront d'un bonus sans avoir fait d'effort.

\section{Les économies d'eau remises en cause}

Les irrigants ont souhaité souligner l'absurdité de l'incitation. Il est difficile d'imaginer un irrigant vouloir économiser de l'eau : «Si j'ai trop d'eau, j'irrigue plus de surface ! » ou bien « Je préfère donner l'eau à mon voisin plutôt que de ne pas l'utiliser ». D'autres jugent peu probable qu'un irrigant choisisse de recevoir une subvention plutôt que d'irriguer : «Produire, c'est son métier. Ce système [de bonus-malus], ça équivaut à accepter de ne pas faire son métier. C'est difficile ». Ces citations peuvent porter plusieurs sens. Les irrigants remettent-ils en cause le fait même d'abandonner l'irrigation, considérant cet engagement comme un changement radical d'orientation technique ? Est-ce plutôt par contrainte économique, et donc face à un montant de bonus proposé trop faible, qu'ils tiennent ce discours ? Ou bien cela relève-t-il d'un discours stratégique ?

\section{Une opposition de principe}

Les participants ont également montré une opposition face au principe de « la carotte et du bâton », qu'ils jugent dévalorisant. Ils rejettent également une monétarisation du système. Ils proposent quelques améliorations : retirer le droit de prélever aux irrigants qui récidivent ; imposer des sanctions en volume applicables immédiatement; proposer un montant du bonus qui soit supérieur de manière certaine au coût de la perte induite par l'économie d'eau.

\section{Le contrat solidaire Efficace sous certaines conditions}

Les participants ont semblé très favorables au contrat solidaire, $90 \%$ d'entre eux l'estimant souhaitable. Il est d'ailleurs préféré parmi tous ceux proposés, rassemblant l'adhésion de 27 des 30 participants.

L'idée de solidarité a plu : «Le côté solidaire, ça va dans le bon sens »; « La notion de solidarité, ici on est plutôt pour ». Les idées de responsabilisation ainsi que d'engagement mutuel ont été également appréciées : pour $93 \%$ des participants, l'engagement dans un contrat solidaire peut permettre de faire respecter le volume prélevable. L'image publique de bon gestionnaire de l'eau, la réduction de la pression individuelle exercée par l'Administration et l'abaissement du risque de dépassement et de l'incertitude sur le comportement des autres ont également été cités comme des avantages du contrat. Ces raisons expliquent que $85 \%$ des irrigants se disent prêts à signer un tel contrat. Ils sont 12 sur 15 parmi les irrigants de l'ADIV et 5 sur 5 parmi les irrigants du Bé.

\section{Une démarche volontaire}

Certains participants ont estimé que pour être vraiment efficace, la participation de tous les irrigants d'un même secteur devrait être obligatoire. À défaut, l'irrigant qui reste en individuel pourrait dépasser son volume prélevable, jusqu'à déclencher la coupure et réduire à néant les efforts du groupe. D'autres, au contraire, insistent sur la nécessité de conserver le principe de libre participation. Ils mettent en garde contre cet « amalgame », considérant qu'un irrigant qui ne signe pas n'est pas forcément «le vilain petit canard». Selon 
eux, une démarche volontaire garantit la bonne volonté et l'engagement des signataires.

\section{La sanction collective majorée :} nécessaire ou anti productive?

La majoration de la pénalité a été reçue différemment selon les groupes : les irrigants des sources du Bé la jugent recevable pourvu qu'elle soit compensée par une minoration conséquente de la cotisation ; le groupe des irrigants de l'ADIV l'a immédiatement rejetée : elle serait suffisante à dissuader l'irrigant de signer un contrat, l'incertitude sur le comportement des autres étant trop forte. Ce groupe estime que dans ces conditions, $18 \%$ des irrigants signeront un contrat, contre $63 \%$ si la pénalité n'est pas majorée. Pour certains, une amende collective suffit à inciter les irrigants à respecter le volume attribué global. Ces différences de réponse révèlent une divergence des deux groupes dans leur façon de considérer le contrat solidaire et plus certainement dans la confiance qu'ils placent dans leurs voisins ou collègues. L'adoption et la réussite de l'instrument dépendraient donc des relations internes aux groupes.

\section{Un instrument à ne pas envisager sur des secteurs sensibles}

Les participants soulignent une autre difficulté d'application du contrat dans le cas du Clain : sur des indicateurs sensibles aux prélèvements l'incertitude sur la ressource et sur le comportement des autres sont tels que la solidarité peinera à primer sur la méfiance dans les relations entre irrigants.

\section{Une réduction de cotisation vraiment indispensable?}

Le débat a très peu fait allusion à la réduction de la cotisation, comme si elle n'était pas déterminante dans le choix de signer un contrat. Les irrigants s'étant montrés immédiatement enthousiasmés par le contrat solidaire : les avantages qu'il offre suffiraient à inciter les irrigants à signer. On peut alors penser que la réduction sur la cotisation n'aurait qu'un effet d'aubaine.

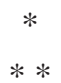

En nous rattachant au contexte de mise en place des OUGC, nous avons développé des instruments de gestion appliqués à un niveau décentralisé. Le bassin du Clain, dans la Vienne, a été choisi pour tester ces instruments de manière qualitative. Les ateliers ont démontré un réel intérêt et une bonne capacité d'analyse et de réflexion prospective des acteurs du terrain. Le premier instrument, basé sur la redistribution, aux irrigants économes, de la recette de pénalités individuelles (malus) sous forme de récompenses financières (bonus) a été jugé peu désirable. La complexité du système, l'incertitude sur le montant des bonus et plus globalement la dimension monétaire du système n'ont pas convaincu les participants de son efficacité à faire respecter le volume prélevable.

Le second scénario, le contrat solidaire, présentait un scénario d'autorégulation imbriquée. Celui-ci a été jugé souhaitable par $90 \%$ des participants qui ont apprécié ses avantages opérationnels : allègement de la pression de l'administration, réduction des risques de coupure, réduction des incertitudes sur le comportement des autres, engagement réciproque envers les autres irrigants.

Les irrigants ont évalué très positivement la démarche méthodologique : le recours à des scénarios narratifs présentés sous forme d'articles de presse a été apprécié ; leur ancrage sur un terrain réel s'est également avéré pertinent. D'après les irrigants, les débats générés par les ateliers ont nourri leurs réflexions personnelles sur la gestion future de l'eau dans leurs exploitations. Ces indications ont pu leur fournir des pistes de réflexion pour élaborer d'un règlement intérieur pour le futur OUGC, mais également pour identifier les 
RECHERCHES

principes de gestion qui leur paraissent fondamentaux de respecter.

Ce travail a mis en évidence l'influence du contexte sur l'opérationnalité et l'efficacité d'un instrument de gestion. Dans le bassin du Clain, le type d'exploitation agricole, la forte réactivité de la nappe et la préexistence d'une structuration des irrigants semblent déterminants. Les scénarios doivent désormais être discutés dans d'autres terrains en France, contrastant avec le bassin du Clain de par leur contexte hydrogéologique, agricole ou institutionnel. Une fois testés qualitativement, les instruments de gestion nécessiteront un test quantitatif, en utilisant l'économie expérimentale. Ces différentes méthodes permettront d'identifier d'autres facteurs contextuels influant sur la réussite d'un système de gestion, et ainsi de produire des recommandations de politiques publiques qui soient adaptées aux contextes locaux.

Les auteurs remercient les acteurs du bassin du Clain pour leur disponibilité et la qualité des échanges qui ont eu lieu au cours des ateliers. Nous remercions également Cécile Hérivaux (Brgm) pour sa participation à l'organisation des ateliers, ainsi que le programme Carnot de l'ANR, l'Irstea et le Brgm pour le financement de la thèse préparée par la première auteure de cet article.

\section{RÉFÉRENCES BIBLIOGRAPHIQUES}

Agrawal A. (2001). Common property institutions and sustainable governance of resources. World Development, vol. 10, n²9, p. 1649-1672.

Aoki M., Challe E. (2006). Fondements d'une analyse institutionnelle comparée. Paris, Albin Michel, 608 p.

Baland J.-M., Platteau J.-P. (1996). Halting Degradation of Natural Resources: Is There a Role for Rural Communities? , Food and Agriculture Organisation, $423 \mathrm{p}$.

Bourgeois P. (2011). Organismes uniques de gestion collective. Cemagref, La gestion collective de la ressource en eau pour l'irrigation et les Associations Syndicales Autorisées, Montpellier, 8 novembre, 45 p.

Conseil général de Vienne (2011). SAGE Clain - État initial. 265 p.

Fehr E., Gächter S. (2000). Cooperation and Punishment in Public Good Experiments. The American Economic Review, vol. 4, $\mathrm{n}^{\circ}$ 90, p. 980-993.

Figureau A.-G., avec la collaboration de Montginoul M. et Rinaudo J.-D. (2012). Gestion quantitative de l'eau d'irrigation en France : bilan de l'application de la loi sur l'eau et les milieux aquatiques de 2006.
$\mathrm{N}^{\circ}$ BRGM/RP-61626-FR, 50 p. En ligne : http://pmb.brgm.fr/brgm/brgm_broogle_ notice.php?id=144878.

Gleyses G., Rieu T. (2004). L'irrigation en France. État des Lieux 2000 et évolution. Cemagref, $60 \mathrm{p}$.

Hardin G. (1968). The Tragedy of the Commons. Science, $\mathrm{n}^{\circ}$ 162, p. 1243-1248.

Koundouri P. (2004). Current issues in the economics of groundwater resource management Journal of Economic Surveys, vol. 5, $\mathrm{n}^{\circ} 18$, p. 703-740.

Lafitte J.-J., Devos P., Portet P. (2008). Les Organismes Uniques d'Irrigation. 78 p.

Llamas M. R., Martinez-Santos P. (2005). Intensive Groundwater USe: Silent Revolution and Potential Source of Conflicts. Journal of Water Resources Planning and Management, sept.-oct., p. 337-341.

Loi (1984). $\mathrm{N}^{\circ} 84-512$ du 29 juin relative à la pêche en eau douce et à la gestion des ressources piscicoles. Journal Officiel de la République française, $\mathrm{n}^{\circ} 30$, juin 1984, p. 2039-2045

Loi (2006). $\mathrm{N}^{\circ} 2006-1772$ du 30 décembre sur l'eau et les milieux aquatiques. Journal 
Officiel de la République française, $\mathrm{n}^{\circ} 303$, 31 décembre 2006, texte $n^{\circ}$ 3/175.

Lopez-Gunn E. (2003). The Role of Collective Action in Water Governance: A Comparative Study of Groundwater User Associations in La Mancha Aquifers in Spain. Water International, vol. $3, \mathrm{n}^{\circ} 28$, p. 367-378.

Lopez-Gunn E., Martinez-Cortina L. (2006). Is self-regulation a myth? Case study on Spanish groundwater user associations and the role of higher-level authorities. Hydrogeology Journal, n ${ }^{\circ}$ 14, p. 361-379.

Ministère de l'Écologie, du Développement et de l'Aménagement Durables (2007). Décret n²007-1381 du 24 septembre 2007 relatif à l'organisme unique chargé de la gestion collective des prélèvements d'eau pour l'irrigation. Journal Officiel de la République française, $\mathrm{n}^{\circ} 223,26$ septembre, texte 4/11.

Ministère de l'Écologie, de l'Énergie, du Développement Durable et de l'Aménagement du Territoire (2008). Circulaire du 30 juin 2008 relative à la résorption des déficits quantitatifs en matière de prélèvement d'eau et gestion collective des prélèvements d'irrigation. Bulletin Officiel du MEEDDAT, $\mathrm{n}^{\circ} 15$, texte $7 / 21,20 \mathrm{p}$.

Ministère de l'Écologie, du Développement Durable, des Transports et du Logement (2012). Décret $n^{\circ}$ 2012-84 relatif à la participation financière des préleveurs irrigants aux dépenses liées aux missions de l'Organisme unique de gestion collective des prélèvements en eau pour l'irrigation, Journal Officiel de la République Française, ${ }^{\circ} 22$, 26 janvier, texte $5 / 115$.

Morice E. (2012), État des lieux de la mise en place des OUGC [entretien]. Communication personnelle.

Onema, BRGM (2011). Banque nationale des prélèvements quantitatifs en eau (BNPE). En ligne : http://bnpe.brgm-rec.fr/ (consulté le 3 septembre 2012).

Ostrom E. (1990). Governing the Commons: The Evolution of Institutions for Collective Action. Cambridge University Press, 298 p.

Ostrom E., Gardner R. (1993). Coping with Asymmetries in the Commons: SelfGoverning Irriagtion Systems Can Work. Journal of Economic Perspectives, vol. 4, $\mathrm{n}^{\circ}$ 7, p. 93-112.
Ostrom, E. (2000). Collective Action \& the Evolution of Social Norms. Journal of Economic Perspectives, vol. 3, $\mathrm{n}^{\circ} 14$, p. 137-158.

Parlement européen et Conseil de l'Union européenne (2000). Directive 2000/60/CE du 23 octobre 2000 établissant un cadre pour une politique communautaire dans le domaine de l'eau. Journal Officiel des Communautés européennes, $\mathrm{n}^{\circ} \mathrm{L} 327$, p. 1-72.

Rinaudo J. D., Montginoul M., Varanda M., Bento S. (2012) Envisioning innovative groundwater regulation policies through scenario workshops in France and Portugal. Irrigation and Drainage, vol. 61, p. 65-74.

Romagny B., Riaux J. (2007). Communitybased agricultural water management in the light of participative policies: A cross-cultural look at cases in Tunisia and Morocco. Regards croisés Tunisie/Maroc, vol. 6, $\mathrm{n}^{\circ}$ 52, p. 1179-1196.

Rose C. M. (2002). Common Property, Regulatory Property and Environmental Protection: Comparing Community-Based Management to Tradable Environmental Allowances. In The Drama of the Commons, National Academy Press: Washington, DC, 233-257 p.

Ruf T. (2011). Le façonnage des institutions d'irrigation au $\mathrm{XX}^{\mathrm{e}}$ siècle, selon les principes d'Elinor Ostrom, est-il encore pertinent en 2010?, Natures Sciences Sociétés, vol. $4, n^{\circ} 19$, p. 395-404.

Salzman J. (2005). Creating Markets for Economic Services: Notes from the field. New York University Law Review, vol. 600, $\mathrm{n}^{\circ} 80, \mathrm{p} .101-184$.

Schlager E., Lopez-Gunn E. (2005). Collective systems for water management: is the Tragedy of the Commons a myth? In Water Crisis: Myth or Reality? Taylor and Francis, p. 44-58.

Sefton M., Shupp R.,Walker J. M. (2007). The effect of rewards and sanctions in provision of public goods. Economic Inquiry, vol. 4, $\mathrm{n}^{\circ} 45$, p. 671-690.

Segerson K. (1988). Uncertainty and Incentives for NonPoint Pollution Control. Journal of Environmental Economics and Management, $\mathrm{n}^{\circ} 15$, p. $87-98$. 
Theesfeld I. (2010). Insitutional Challenges for National Groundwater Governance: Policies and Issues. Ground Water, $\mathrm{n}^{\circ} 48,1$, p. 131-142.

Van Steenbergen F. (2006). Promoting local management in groundwater. Hydrogeology Journal, ${ }^{\circ} 14$, p. 380-391.
Vyrastekova J., von Soest D. (2003). Centralized Common-Pool Management and Local Community Participation. Land Economics, vol. $4, n^{\circ} 79$, p. 500-514.

\section{ANNEXE}

Annexe 1. Exemple d'article envoyé aux participants

\section{Le Poitou Rural}

\section{ÉVÉNEMENT}

17 octobre 2020

Nouvelles règles de gestion des volumes d'irrigation

Au troisième jour des assises de l'eau, l'Organisme de Répartition des Volumes d'eau d'Irrigation du Clain (ORVIC) a présenté son projet de nouveau règlement intérieur. Désormais, les agriculteurs qui dépassent leur attribution devront payer une pénalité financière. Ceux qui, au contraire, parviennent à consommer moins seront récompensés.

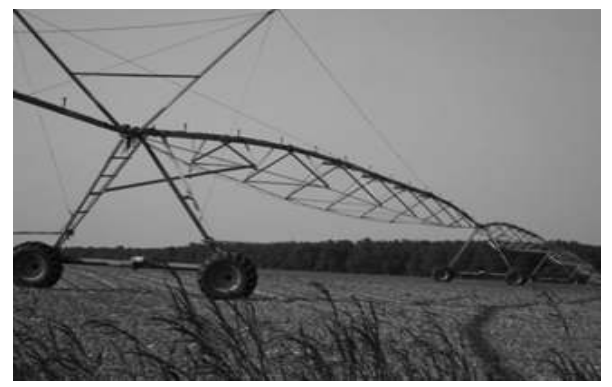

Le constat était partagé par la profession agricole et les représentants de l'État : mettre un gendarme derrière chaque agriculteur n'est pas une solution. Pourtant l'ORVIC doit absolument tenir son engagement vis-à-vis de l'État. Faire en sorte que la totalité des prélèvements de ses membres ne dépasse pas le Volume Prélevable d'Irrigation, tel est le défi que cherche aujourd'hui à relever l'ORVIC.

\section{Un système de bonus - malus}

Jean Barre, président de l'ORVIC, explique très simplement ce qui est proposé. « Comme on ne pourra jamais empêcher que certains agriculteurs dépassent, même occasionnellement, les volumes d'eau qui leur ont été attribués, il faut que d'autres consomment moins que ce à quoi ils ont droit. D'où l'idée d'utiliser à la fois la carotte et le bâton. Le bâton, ce sera une pénalité financière que devront payer à l'ORVIC les agriculteurs qui ne respectent pas leur attribution. Et la carotte, un bonus financier versé à ceux qui réduisent leur consommation. Les économies d'eau réalisées par les uns compenseront ainsi les excès des autres. Et les amendes payées par les uns serviront à récompenser les autres. »

\section{Comment ça marche ?}

La pénalité sera payée proportionnellement au volume du dépassement. Pour qu'elle soit dissuasive, le Conseil d'Administration de l'ORVIC a décidé de l'établir à 30 centimes d'euro par mètre cube. Cette pénalité sera doublée dès que l'irrigant dépassera de plus de $30 \%$ son attribution.

Pour bénéficier d'un bonus, il faudra montrer patte blanche. «La première 
condition est de ne pas avoir été sanctionné l'année précédente. La seconde condition est de ne jamais avoir dépassé de plus de $10 \%$ son Volume hebdomadaire pendant toute la saison d'irrigation. » Si ces deux conditions sont remplies, le bonus sera proportionnel à l'économie d'eau réalisée. La valeur de départ est fixée à 40 centimes du mètre cube.

\section{Proposition juste et crédible}

Pour Jules Fondeau de la Chambre d'Agriculture, «ce système est juste, car il incite tous les agriculteurs à éviter les excès sans pour autant mettre sur la paille ceux qui doivent faire face à un coup dur ; 30 centimes de pénalité, c'est dur, mais supportable. Le doublement de la pénalité appliqué à ceux qui dépassent de plus de $30 \%$ leur quota fera aussi rentrer dans le rang ceux qui prennent systématiquement le risque de dépasser. Le système est également juste parce qu'il rétribue ceux qui font des efforts d'économie d'eau et qui y laissent une partie de leur revenu ».

Des garanties ont également été données au Préfet. Le règlement prévoit que tout agriculteur qui ne paierait pas les pénalités financières sera exclu de l'ORVIC, ce qui le privera du droit de prélever. Une menace suffisamment crédible pour que le système fonctionne.

\section{Le système sera-t-il économiquement viable?}

La principale difficulté que l'ORVIC devra résoudre consistera à assurer l'équilibre financier de ce système : «il s'agit d'un système fermé, dans lequel les sommes payées par certains agriculteurs bénéficient à d'autres, sans aucune entrée financière extérieure » explique Jean Barre. «Le niveau $d u$ bonus sera donc ajusté en fonction $d u$ montant des recettes générées par les malus ainsi que du nombre de bénéficiaires ».

\section{Opposition}

La proposition de l'ORVIC n'a cependant pas fait l'unanimité au sein même de la profession. Pour Anibal Lebret du Syndicat Nouveaux Paysans «on signe un chèque en blanc en autorisant les dépassements à ceux qui peuvent payer. Tout cela accentuera les différences entre les exploitations agricoles, au profit des plus grosses. Il faut durcir les sanctions pénales, les appliquer strictement et tout rentrera vite dans l'ordre». Pour les associations de consommateurs, la gestion de ce système va coûter très cher, avec un risque de contentieux important. Quant au Préfet, il n'a pas encore communiqué sur le projet. 